\title{
Existence d'orbites quasi-periodiques dans les attracteurs de Birkhoff
}

\author{
Patrice Le Calvez \\ Mathematiques, Université Paris-Sud, Bâtiment 425, Centre d'Orsay, \\ F-91405 Orsay Cedex, France
}

\begin{abstract}
For each number $\varrho$ between the lower and the upper rotation number of the Birkhoff attractor of a dissipative monotone twist map, there is a periodic or quasi-periodic orbit with rotation number $\varrho$.
\end{abstract}

On va prouver ici un théorème d'existence d'orbites quasi-périodiques pour un difféomorphisme de l'anneau déviant la verticale et dissipatif. On utilise pour cela une méthode géométrique simple conduisant d'autre part au théorème géométrique de Poincaré-Birkhoff dans le cas de l'itérée d'une application déviant la verticale.

\section{Rappels sur les Attracteurs de Birkhoff}

Les résultats qui suivent se trouvent pour la plupart, mais sous une terminologie différente, dans les articles [B1] et [Cha].

On note $\mathbb{T}^{1}$ le tore de dimension 1 , et on considère $\mathbb{T}^{1} \times \mathbb{R}$ muni de la mesure de Lebesgue et de ses projections canoniques $p_{1}$ et $p_{2}$. On définit de même sur son revêtement $\mathbb{R} \times \mathbb{R}$ les projections $\tilde{p}_{1}$ et $\tilde{p}_{2}$, la translation $\tilde{T}(\tilde{T}(\tilde{\theta}, r)=(\tilde{\theta}+1, r))$ et la projection canonique $\pi: \mathbb{R} \times \mathbb{R} \rightarrow \mathbb{T}^{1} \times \mathbb{R}$.

On notera $x=(\theta, r)$ un point de $\mathbb{T}^{1} \times \mathbb{R}$ et $\tilde{x}=(\tilde{\theta}, r)$ un point de $\mathbb{R} \times \mathbb{R}$.

On écrira respectivement $\bar{A}, \operatorname{Fr}(A), \mathbb{T}^{1} \times \mathbb{R} \backslash A$ pour l'adhérence, la frontière et le complémentaire d'une partie $A$ de $\mathbb{T}^{1} \times \mathbb{R}$ et $\widetilde{A}$ l'ensemble $\pi^{-1}(A)$.

On dit qu'un compact connexe $\Lambda$ de $\mathbb{T}^{1} \times \mathbb{R}$ sépare $\mathbb{T}^{1} \times \mathbb{R}$ si, et seulement si $\mathbb{T}^{1} \times \mathbb{R} \backslash \Lambda$ possède deux composantes connexes non bornées (éventuellement d'autres composantes connexes bornées); on note alors respectivement $U(\Lambda)$ et $V(\Lambda)$ les composantes connexes contenant $\left.\left.\mathbb{T}^{1} \times\right]-\infty,-M\right]$ et $\mathbb{T}^{1} \times[M,+\infty[$ où $M$ est grand. Si $\mathbb{T}^{1} \times \mathbb{R} \backslash \Lambda=U(\Lambda) \cup V(\Lambda)$, on dira que $\Lambda$ est un compact annulaire.

On considère un difféomorphisme $f$ de $\mathbb{T}^{1} \times \mathbb{R}$, de classe $C^{1}$, homotope à l'identité, tel que:

i) Il existe $\alpha \in] 0,1\left[\right.$ tel que, pour tout $x \in \mathbb{T}^{1} \times \mathbb{R}, 0<\operatorname{Det} D f(x)<\alpha$. 
ii) L'application $f$ dévie la verticale à droite: il existe $\beta \in] 0, \pi / 2[$ tel que, pour tout $x \in \mathbb{T}^{1} \times \mathbb{R}$, on ait

$$
D f(x) \cdot(0,1) \in C_{I}(\beta), \quad D f^{-1}(x) \cdot(0,1) \in C_{I I}(\beta),
$$

où $C_{I}(\beta)$ est le cône formé des demi-droites $O D$ vérifiant $-\pi+\beta<$ angle $(O r, O D)$ $<-\beta$, et où $C_{I I}(\beta)=-C_{I}(\beta)$.

Fig. 1.

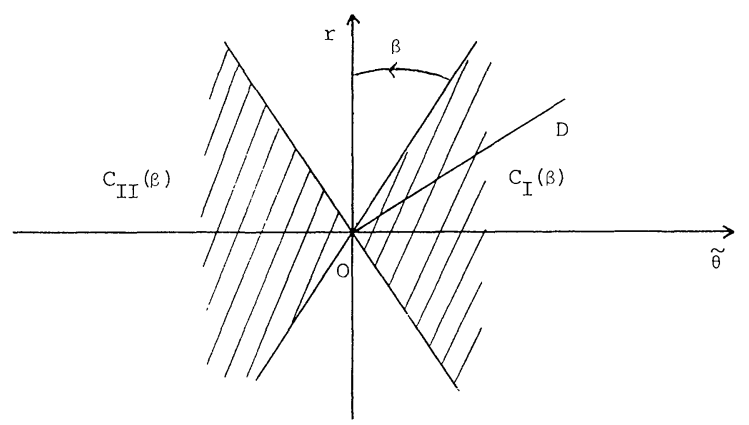

On veut étudier les compacts connexes séparant $\mathbb{T}^{1} \times \mathbb{R}$ invariants par $f$.

On montre d'abord l'existence de tels ensembles. D'après i), si $M>0$, l'aire de $f\left(\mathbb{T}^{1} \times[0, M]\right)$ est plus petite que $\alpha M$. Par conséquent, si $M$ est suffisamment grand, on peut trouver un point $x$ vérifiant: $p_{2}(x) \geqq M+2 \operatorname{cotg} \beta, p_{2}(f(x)) \leqq M$ $-2 \operatorname{cotg} \beta$. Si jamais on a $f\left(\mathbb{T}^{1} \times\{M\}\right) \cap \mathbb{T}^{1} \times\{M\} \neq \emptyset$, il existe alors deux points $\tilde{x}$ et $\tilde{y}$ dans $\mathbb{R} \times \mathbb{R}$ vérifiant, pour un relèvement $\tilde{f}$ de $f$, les relations: $\tilde{p}_{1}(\tilde{y}) \leqq \tilde{p}_{1}(\tilde{x})$ $\left.<\tilde{p}_{1}(\tilde{y})+1, \tilde{p}_{2}(\tilde{f}(\tilde{y}))=\tilde{p}_{2}(\tilde{y})\right)=M, \tilde{p}_{2}(\tilde{x}) \geqq M+2 \operatorname{cotg} \beta, \tilde{p}_{2}(\tilde{f}(\tilde{x})) \leqq M-2 \operatorname{cotg} \beta$, et ceci est impossible par la condition ii). En effet, l'angle entre le vecteur $(0,1)$ et $\tilde{x}-\tilde{T}(\tilde{y})$ serait alors compris entre 0 et $\beta$ et on aurait $\tilde{p}_{1} \circ \tilde{f}(\tilde{x}) \geqq \tilde{p}_{1} \circ \tilde{f}(\tilde{y})+1$. Par conséquent, il existerait $k>1$ tel que l'angle entre $(0,1)$ et $\widetilde{T}^{k} \circ \widetilde{f}(\tilde{y})-\tilde{f}(\tilde{x})$ soit compris entre $-\beta$ et 0 ; on en déduirait alors, par passage à l'inverse, l'inégalité $\tilde{p}_{1}(\tilde{x}) \geqq \tilde{p}_{1}(\tilde{y})+k$ et donc une contradiction. Ainsi, pour $M$ assez grand, on a:

$$
\left.f\left(\mathbb{T}^{1} \times[-M,+M]\right) \subset \mathbb{T}^{1} \times\right]-M,+M[.
$$

Soit maintenant $\Lambda_{1}$ un compact connexe quelconque séparant $\mathbb{T}^{1} \times \mathbb{R}$ et invariant par $f$. Il est de mesure nulle, donc d'intérieur vide, et, de plus, annulaire. On considère alors $\Lambda_{1}^{\prime}=\operatorname{Fr} U\left(\Lambda_{1}\right) \cap \operatorname{Fr} V\left(\Lambda_{1}\right)$, c'est un compact annulaire invariant par $f$, et on montre que $\Lambda_{1}^{\prime}=\operatorname{Fr} U\left(\Lambda_{1}^{\prime}\right)=\operatorname{Fr} V\left(\Lambda_{1}^{\prime}\right)$.

De plus, si $\Lambda_{2}$ est un autre compact annulaire invariant par $f$, on a l'égalité $\Lambda_{2}^{\prime}=\Lambda_{1}^{\prime}$. En effet, l'ouvert $U\left(\Lambda_{1}^{\prime}\right) \cap V\left(\Lambda_{2}^{\prime}\right)$ est borné et invariant par $f$, donc vide. On en déduit $U\left(\Lambda_{1}^{\prime}\right) \cap \overline{V\left(\Lambda_{2}^{\prime}\right)}=\emptyset$ et donc l'inclusion $U\left(\Lambda_{1}^{\prime}\right) \subset U\left(\Lambda_{2}^{\prime}\right)$ qui suffit pour conclure. Il n'y a donc qu'un compact annulaire qui est frontière commune des deux composantes connexes de son complémentaire, invariant par $f$. On l'appelle l'attracteur de Birkhoff de $f$ et on le note $\Lambda^{\prime}$, il est contenu dans tous les compacts annulaires invariants. C'est aussi l'attracteur de Birkhoff des itérées $f^{q}$ de $f$ où $q \in \mathbb{N}^{*}$.

On obtient de la déviation de la verticale une propriété géométrique supplémentaire. Si $\Lambda$ est un compact annulaire invariant par $f$, tout point de $U(\Lambda)$ 
est accessible par un chemin négatif issu verticalement de l'infini à valeur dans $U(\Lambda)$, de même pour tout point de $V(\Lambda)[\mathrm{B} 1, \mathrm{He}]$.

Fig. 2.

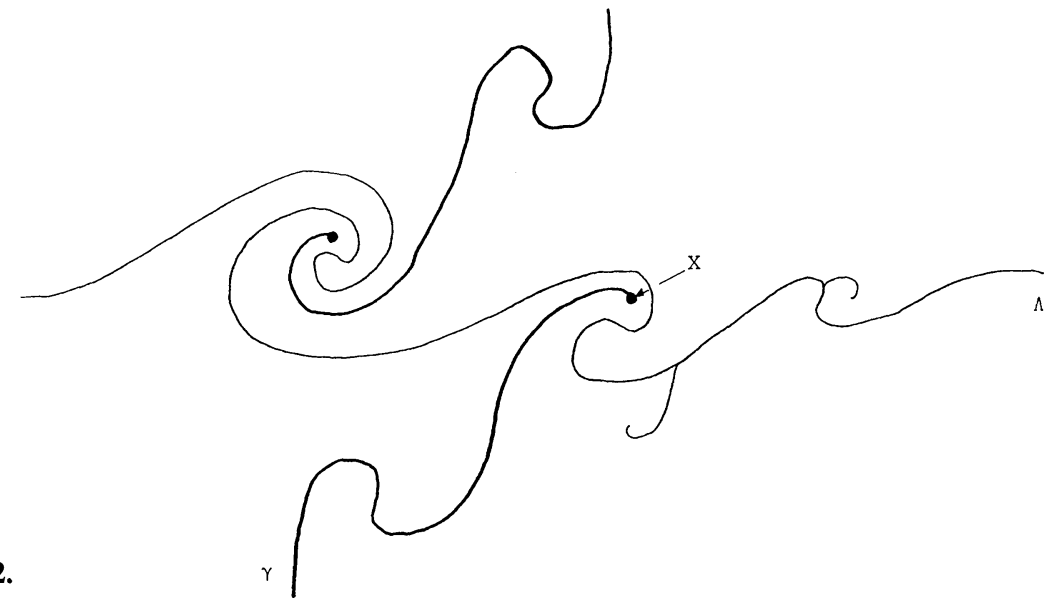

Rappel. Le chemin $\gamma$ est dit négatif si c'est un plongement $C^{1}$ de $\left.]-\infty, 0\right]$ dans $U(\Lambda)$ avec $\gamma(0)=x$ et $\gamma^{\prime}(t)=(0,1)$ si $t \leqq \tau(\tau<0)$. De plus, le relèvement à $\mathbb{R}$ de l'angle que fait le vecteur $(0,1)$ avec la tangente en $\gamma(t)$, et qui vaut 0 quand $t \leqq \tau$, est strictement négatif si $t>\tau$.

On écrit, pour $\theta \in \mathbb{T}^{1}$, respectivement $\left(\theta, u_{\Lambda}^{-}(\theta)\right)$ et $\left(\theta, v_{\Lambda}^{+}(\theta)\right)$ les points de $\Lambda \cap\{\theta\} \times \mathbb{R}$ d'ordonnée minimale et maximale. On note alors respectivement $\Lambda^{-}$, $\Lambda^{+}, \quad U^{-}(\Lambda), \quad V^{+}(\Lambda)$ les graphes de $u_{\Lambda}^{-}$, de $v_{\Lambda}^{+}$et les ensembles $\left\{(\theta, r) \in \mathbb{T}^{1} \times \mathbb{R}, r<u_{\Lambda}^{-}(\theta)\right\},\left\{(\theta, r) \in \mathbb{T}^{1} \times \mathbb{R}, r>v_{\Lambda}^{+}(\theta)\right\}$.

La propriété précédente prouve alors les résultats suivants [B1]:

i) $f^{-1}\left(U^{-}(\Lambda)\right) \subset U^{-}(\Lambda) ; f^{-1}\left(V^{+}(\Lambda)\right) \subset V^{+}(\Lambda)$.

ii) $f^{-1}\left(\overline{\Lambda^{+}}\right) \subset \Lambda^{+} ; f^{-1}\left(\overline{\Lambda^{-}}\right) \subset \Lambda^{-}$.

iii) L'application $u_{\Lambda}^{-}$est semi-continue inférieurement, continue à droite et admet une limite à gauche en tout point.

iv) L'application $v_{\Lambda}^{+}$est semi-continue supérieurement, continue à gauche et admet une limite à droite en tout point.

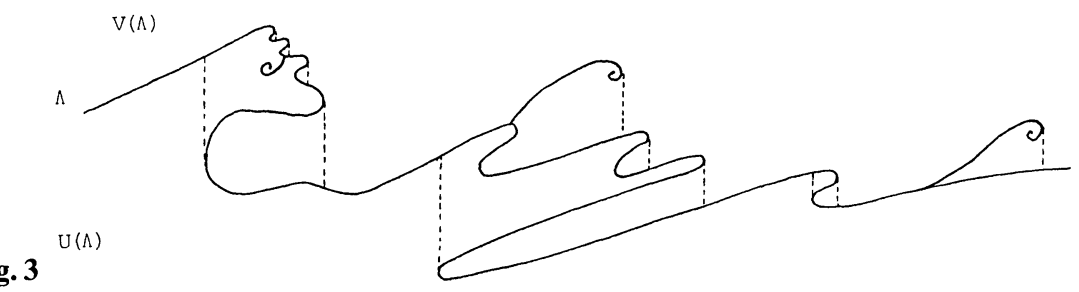

Choisissant un relèvement $\tilde{f}$ de $f$, on montre que $\tilde{f}^{-1}$ préserve sur $\tilde{\Lambda}^{+}$l'ordre donné par la première projection et on en déduit l'existence d'un réel $\tilde{\varrho}_{\Lambda}^{+}$tel que, pour tout $\tilde{x} \in \tilde{\Lambda}^{+}$,

$$
\lim _{n \rightarrow+\infty} \frac{\tilde{p}_{1}\left(\tilde{f}^{-n}(\tilde{x})\right)}{n}=-\tilde{\varrho}_{\Lambda}^{+},
$$

puis d'un ensemble d'Aubry-Mather dans $\Lambda^{+}$, de nombre de rotation $\tilde{\varrho}_{\Lambda}^{+}$. 
Note ([Che]). Un ensemble d'Aubry-Mather est un compact $\Xi$ de $\mathbb{T}^{1} \times \mathbb{R}$ invariant par $f$ et minimal, s'envoyant injectivement sur $\mathbb{T}^{1}$ par la projection $p_{1}$, et tel que l'ordre ainsi défini sur $\widetilde{\Xi}$ est conservé par $\tilde{f}$. Il existe alors un réel $\varrho$ appelé nombre de rotation, et tel que pour tout $\tilde{x} \in \widetilde{\Xi}$,

$$
\lim _{n \rightarrow \pm \infty} \frac{\tilde{p}_{1}\left(\tilde{f}^{n}(\tilde{x})\right)}{n}=\tilde{\varrho} .
$$

On définit de même $\tilde{\varrho}_{\Lambda}^{-}$pour les points radialement accessibles par le bas et, utilisant le fait que $\tilde{f}^{-1}$ dévie la verticale et préserve sur $\tilde{\Lambda}^{+}$et $\tilde{\Lambda}^{-}$l'ordre donné par la première projection, on obtient alors les doubles inégalités suivantes, pour tout $\tilde{x} \in \tilde{\Lambda}$ et pour tout $k \in \mathbb{N}$ :

$$
\begin{aligned}
& -1-k \tilde{\varrho}_{\Lambda}^{+} \leqq \tilde{p}_{1}\left(\tilde{f}^{-k}(\tilde{x})\right)-\tilde{p}_{1}(\tilde{x}) \leqq 1-k \tilde{\varrho}_{A}^{-}, \\
& -1+k \tilde{\varrho}_{\Lambda}^{-} \leqq \tilde{p}_{1}\left(\tilde{f}^{k}(\tilde{x})\right)-\tilde{p}_{1}(\tilde{x}) \leqq 1+k \tilde{\varrho}_{\Lambda}^{+} .
\end{aligned}
$$

On en déduit, si $\Lambda_{1} \subset \Lambda_{2}$ sont deux compacts connexes invariants par $f$, séparant $\mathbb{T}^{1} \times \mathbb{R}$, les inégalités:

$$
\left.\tilde{\varrho}_{\Lambda_{2}}^{-} \leqq \tilde{\varrho}_{\Lambda_{1}}^{-} \leqq \tilde{\varrho}_{\Lambda_{1}}^{+} \leqq \tilde{\varrho}_{\Lambda_{2}}^{+} \quad \text { (en particulier } \tilde{\varrho}_{A}^{-} \leqq \tilde{\varrho}_{\Lambda^{\prime}}^{-} \leqq \tilde{\varrho}_{\Lambda^{\prime}}^{+} \leqq \tilde{\varrho}_{A}^{+}\right) \text {. }
$$

Les nombres $\tilde{\varrho}_{A}^{-}$et $\tilde{\varrho}_{A}^{+}$sont appelés nombres de rotation inférieur et supérieur de $\Lambda$. On écrira plus simplement respectivement $\tilde{\varrho}^{-}, \varrho^{+}, u^{-}, v^{+}$pour $\tilde{\varrho}_{\Lambda^{\prime}}^{-}, \tilde{\varrho}_{\Lambda^{\prime}}^{+}, u_{\Lambda^{\prime}}^{-}, v_{\Lambda^{\prime}}^{+}$.

Il existe une autre interprétation de $\tilde{\varrho}^{-}$et $\tilde{\varrho}^{+}$en termes de points accessibles et non plus seulement radialement accessibles. La théorie de Carathéodory prouve l'existence d'un homéomorphisme $\left.\tilde{H}: \widetilde{U}\left(\Lambda^{\prime}\right) \rightarrow \mathbb{R} \times\right]-\infty, 0[$, commutant avec $\widetilde{T}$, tel que, si $\gamma:\left[0,1\left[\rightarrow \widetilde{U}\left(\Lambda^{\prime}\right)\right.\right.$ est un chemin continu avec $\lim _{t \rightarrow 1} \gamma(t) \in \tilde{\Lambda}^{\prime}$, alors $\tilde{H} \circ \gamma$ admet une limite quand $t \rightarrow 1$ vers un point de $\mathbb{R} \times\{0\}$ ne dépendant que de $\lim _{t \rightarrow 1} \gamma(t)$ [car $\left.\Lambda^{\prime}=\operatorname{Fr} U\left(\Lambda^{\prime}\right)=\operatorname{Fr} V\left(\Lambda^{\prime}\right)\right]$. On obtient ainsi une bijection $\tilde{\eta}$ entre l'ensemble, invariant $\operatorname{par} \tilde{f}$, des points de $\tilde{\Lambda}^{\prime}$ accessibles par le bas et une partie dense $\tilde{\Sigma}$ de $\mathbb{R} \times\{0\}$. On considère alors la bijection $\tilde{\Phi}=\tilde{\eta} \circ \tilde{f} \circ \tilde{\eta}^{-1}$ de $\tilde{\Sigma}$ sur elle-même, elle se prolonge en un homéomorphisme croissant de $\mathbb{R} \times\{0\}$ commutant avec $\widetilde{T}$, le nombre de rotation de cet homéomorphisme est alors égal à $\varrho^{-}$.

C'est d'ailleurs cette interprétation de $\varrho^{-}$qui permet de montrer le caractère topologiquement compliqué de $\Lambda^{\prime}$ dès que $\tilde{\varrho}^{-} \neq \varrho^{+}$. Il ne s'écrit pas comme réunion de deux compacts connexes propres: c'est un continu indécomposable [Cha].

Exemple de Birkhoff. Originellement, Birkhoff donne l'exemple d'un attracteur indécomposable de la façon suivante: soit $f_{0}$ un difféomorphisme de $\mathbb{T}^{1} \times \mathbb{R}$, de classe $C^{1}$, homotope à l'identité, préservant la mesure, déviant la verticale à droite et ayant une zone d'instabilité $A[\mathrm{~B} 2, \mathrm{He}]$ délimitée par deux graphes $C^{-}=g r \psi^{-}$ et $C^{+}=g r \psi^{+}\left(\psi^{-}<\psi^{+}\right)$. On sait que les nombres de rotations $\varrho_{0}^{-}$et $\tilde{\varrho}_{0}^{+}$sur $\widetilde{C}^{-}$et $\widetilde{C}^{+}$d'un relèvement $\widetilde{f}_{0}$ de $f_{0}$ sont distincts. On peut définir alors une famille de difféomorphismes dissipatifs $\left.f_{\varepsilon}, \varepsilon \in\right] 0,1\left[\right.$, en choisissant $\psi$ de classe $C^{1}$, avec $\psi^{-}<\psi<\psi^{+}$, et en posant:

$$
f_{\varepsilon}(\theta, r)=f_{0}(\theta,(1-\varepsilon) r+\varepsilon \psi(\theta)) ;
$$

l'attracteur de Birkhoff de $f_{\varepsilon}$ est alors contenu dans $A$ et ses nombres de rotations inférieurs et supérieurs convergent, pour le relèvement naturel, quand $\varepsilon$ tend vers zéro, respectivement vers $\varrho_{0}^{-}$et $\tilde{\varrho}_{0}^{+}$. Il est indécomposable dès que $\varepsilon$ est assez petit. 


\section{Existence d'Ensembles d'Aubry-Mather}

On garde les notations de l'exemple de Birkhoff. On sait qu'il existe, pour chaque $\tilde{\varrho} \in\left[\tilde{\varrho}_{0}^{-}, \tilde{\varrho}_{0}^{+}\right]$, un ensemble d'Aubry-Mather pour $f_{0}$ dans $A$ de nombre de rotation $\varrho$ (cf. [Che] et réf. cit.). On va montrer le résultat analogue suivant (dans le cas dissipatif), en prenant une application $f$ définie au $\S \mathrm{I}$.

Théorème. Pour tout nombre $\tilde{\varrho} \in\left[\tilde{\varrho}^{-}, \tilde{\varrho}^{+}\right]$, il existe dans $\Lambda^{\prime}$ un ensemble d'AubryMather pour $\tilde{f}$ de nombre de rotation $\tilde{\varrho}$.

On suppose que $p \in \mathbb{Z}, q \in \mathbb{N}^{*}$, et que $p$ et $q$ sont premiers entre eux. On rappelle qu'un point $\frac{p}{q}$-périodique de $\tilde{f}$ est un point $\tilde{x} \in \mathbb{R} \times \mathbb{R}$ vérifiant $\tilde{f}^{q}(\tilde{x})=\widetilde{T}^{p}(\tilde{x})$. En particulier, tout ensemble d'Aubry-Mather de nombre de rotation $\frac{p}{q}$ est l'orbite d'un point $x=\pi(\tilde{x})$, où $\tilde{x}$ est $\frac{p}{q}$-périodique.

On montre d'abord les résultats suivants:

Proposition 1. Pour tout $\left.\frac{p}{q} \in\right] \tilde{\varrho}^{-}, \tilde{\varrho}^{+}\left[\right.$, il existe un point $\frac{p}{q}$ périodique dans $\mathbb{R} \times \mathbb{R}$. Proposition 2. Si $\tilde{x} \in \mathbb{R} \times \mathbb{R}$ est un point $\frac{p}{q}$-périodique, avec $\tilde{\varrho}^{-}<\frac{p}{q}<\tilde{\varrho}^{+}$, alors
$\tilde{x} \in \tilde{\Lambda}^{\prime}$ $\tilde{x} \in \tilde{\Lambda}^{\prime}$.

Chacune de ces propositions utilise le lemme suivant (voir §I pour les notations).

Lemme 1. Si $\tilde{y}_{0}=\left(\tilde{\theta}_{0}, r_{0}\right) \in \tilde{U}^{-}\left(\Lambda^{\prime}\right)$ et si $\tilde{x}_{0}=\left(\tilde{\theta}_{0}, u^{-}\left(\tilde{\theta}_{0}\right)\right)$, alors, pour tout $k \in \mathbb{N}$, $\tilde{p}_{1}\left(\tilde{f}^{-k}\left(\tilde{y}_{0}\right)\right)>\tilde{p}_{1}\left(\tilde{f}^{-k}\left(\tilde{x}_{0}\right)\right)$.

Démonstration du Lemme 1. On rappelle que $\tilde{f}^{-1}\left(\tilde{U}^{-}\left(\Lambda^{\prime}\right)\right) \subset \tilde{U}^{-}\left(\Lambda^{\prime}\right)$ et que la restriction de $\tilde{f}^{-1}$ à $\tilde{\Lambda}^{\prime-}$ est croissante. Si l'on pose, pour $k \in \mathbb{N}, \tilde{f}^{-k}\left(\tilde{y}_{0}\right)=\left(\widetilde{\theta}_{k}, r_{k}\right)$, $\tilde{x}_{k}=\left(\tilde{\theta}_{k}, u^{-}\left(\tilde{\theta}_{k}\right)\right)$; la déviation à droite et les résultats mentionnés entraînent les inégalités suivantes:

$$
\begin{aligned}
& \tilde{p}_{1}\left(\tilde{f}^{-1}\left(\tilde{y}_{0}\right)\right)>\tilde{p}_{1}\left(\tilde{f}^{-1}\left(\tilde{x}_{0}\right)\right) \\
& \tilde{p}_{1}\left(\tilde{f}^{-2}\left(\tilde{y}_{0}\right)\right)>\tilde{p}_{1}\left(\tilde{f}^{-1}\left(\tilde{x}_{1}\right)\right)>\tilde{p}_{1}\left(\tilde{f}^{-2}\left(\tilde{x}_{0}\right)\right) \\
& \vdots \\
& \tilde{p}_{1}\left(\tilde{f}^{-k}\left(\tilde{y}_{0}\right)\right)>\tilde{p}_{1}\left(\tilde{f}^{-1}\left(\tilde{x}_{k-1}\right)\right)>\tilde{p}_{1}\left(\tilde{f}^{-2}\left(\tilde{x}_{k-2}\right)\right)>\ldots>\tilde{p}_{1}\left(\tilde{f}^{-k}\left(\tilde{x}_{0}\right)\right) .
\end{aligned}
$$

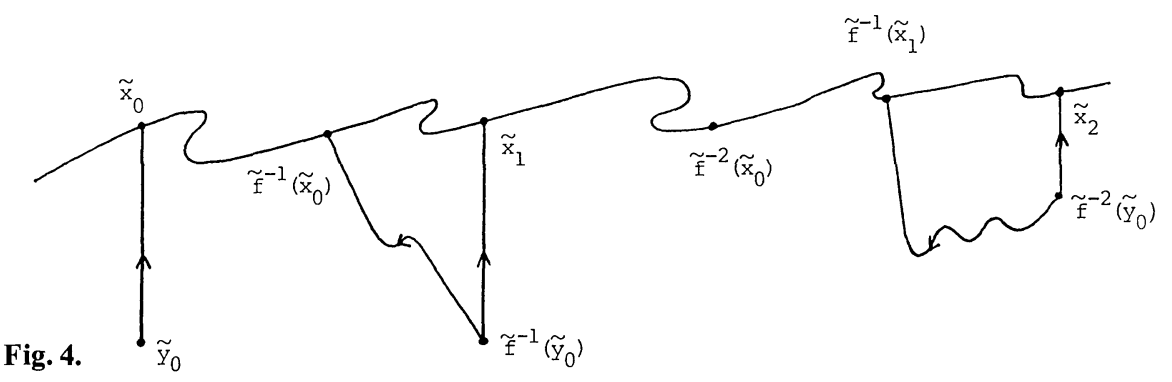


Démonstration de la Proposition 1. La démonstration repose sur l'étude du compact $K$ de $\mathbb{T}^{1} \times \mathbb{R}$ défini par:

$$
K=\left\{x \in \mathbb{T}^{1} \times \mathbb{R} \mid \tilde{p}_{1}\left(\tilde{f}^{q}(\tilde{x})\right)=\tilde{p}_{1}(\tilde{x})+p, \text { où } \pi(\tilde{x})=x\right\},
$$

et plus spécialement sur le résultat suivant:

Lemme 2. Si $L \subset K$ est un compact connexe séparant $\mathbb{T}^{1} \times \mathbb{R}$, il existe un point $\frac{p}{q}$-périodique dans $L$.

Démonstration $d u$ lemme 2 . On considère un point $\tilde{\theta}$ de $\mathbb{R}$, un entier $q \geqq 1$ et le chemin $\mu_{q}$ défini par $\mu_{q}(t)=\tilde{f}^{q}(\tilde{\theta}, t)$ où $t \in \mathbb{R}$. Utilisant la déviation de la verticale vers la droite et le fait que $f$ est un difféomorphisme, on en déduit les égalités:

$$
\begin{aligned}
& \lim _{t \rightarrow+\infty} \tilde{p}_{2} \circ \mu_{q}(t)=\lim _{t \rightarrow+\infty} \tilde{p}_{1} \circ \mu_{q}(t)=+\infty, \\
& \lim _{t \rightarrow-\infty} \tilde{p}_{2} \circ \mu_{q}(t)=\lim _{t \rightarrow-\infty} \tilde{p}_{1} \circ \mu_{q}(t)=-\infty .
\end{aligned}
$$

On peut alors montrer par une récurrence sur $q$ le résultat suivant vérifié pour les chemins négatifs [He]:

le premier et le dernier point de rencontre du chemin $\mu_{q}$ avec une verticale $\left\{\tilde{\theta}^{\prime}\right\} \times \mathbb{R}, \tilde{\theta^{\prime}} \in \mathbb{R}$ (ce qui a un sens d'après ce qui précède) sont les points de rencontre d'ordonnée respectivement maximale et minimale.

Fig. 5.

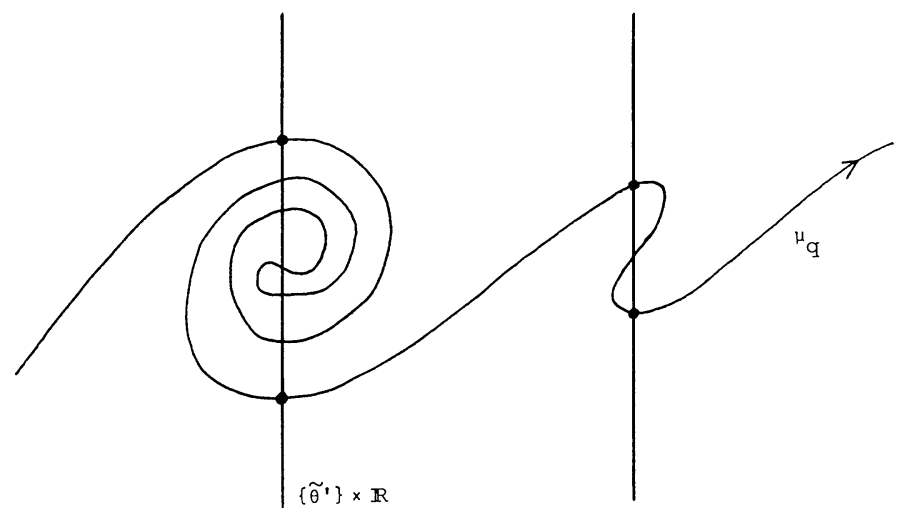

Le résultat est évident quand $q=1$. On suppose le résultat vrai jusqu'au rang $q-1$ et on écrit $\tilde{x}_{0}=\left(\widetilde{\theta}_{0}^{\prime}, r_{0}\right)=\mu_{q}\left(t_{0}\right)$ le premier point de rencontre de $\mu_{q}$ avec la verticale $\left\{\theta_{0}^{\prime}\right\} \times \mathbb{R}$. On note alors $\tilde{x}_{1}=\left(\tilde{\theta}_{1}^{\prime}, r_{1}\right)$ le point $\mu_{q-1}\left(t_{0}\right)=\tilde{f}^{-1}\left(\tilde{x}_{0}\right)$. Le fait que

$$
\mu_{q-1}(]-\infty, t_{0}[) \cap \tilde{f}^{-1}\left(\left\{\tilde{\theta}_{0}^{\prime}\right\} \times\left[r_{0},+\infty[)=\emptyset,\right.\right.
$$

et la propriété appliquée au chemin $\mu_{q-1}$ prouvent alors que $\mu_{q-1}(]-\infty, t_{0}[)$ se trouve à gauche de $\left\{\theta_{1}^{\prime}\right\} \times \mathbb{R}$ et que $\mu_{q-1}$ ne rencontre pas la verticale $\left.\left\{\theta_{1}^{\prime}\right\} \times\right] r_{1},+\infty[$.

En considérant l'image de $\mu_{q-1}(]-\infty, t_{0}[) \cup\left(\left\{\theta_{1}^{\prime}\right\} \times\left[r_{1},+\infty[)\right.\right.$ par $\tilde{f}$, on en déduit que $\mu_{q}$ ne rencontre pas $] r_{0},+\infty[$. 

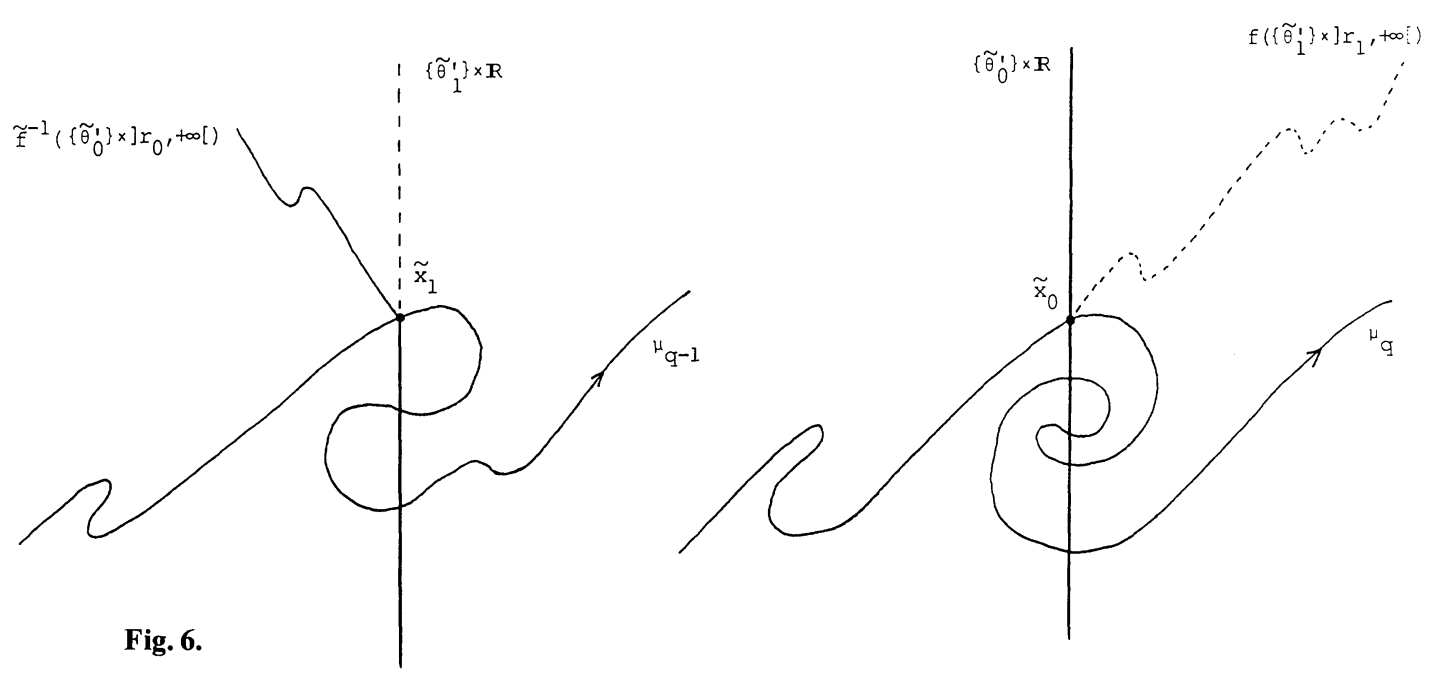

La démonstration est identique pour le dernier point de rencontre.

Si $\tilde{\theta} \in \mathbb{R}$, on note $h^{-}(\widetilde{\theta})$ et $h^{+}(\widetilde{\theta})$ les ordonnées respectivement minimale et maximale de l'ensemble $\{\widetilde{\theta}\} \times \mathbb{R} \cap \widetilde{K}$, on obtient alors des fonctions de période 1 , $h^{-}$et $h^{+}, h^{-} \leqq h^{+}$, vérifiant:

$Q\left\{\right.$ i) Pour tout $\tilde{\theta} \in \mathbb{R}, \tilde{p}_{1}\left(\tilde{f}^{q}\left(\tilde{\theta}, h^{-}(\tilde{\theta})\right)\right)=\tilde{p}_{1}\left(\tilde{f}^{q}\left(\tilde{\theta}, h^{+}(\tilde{\theta})\right)\right)=\tilde{\theta}+p$.

(ii) Pour tout $(\tilde{\theta}, r) \in \mathbb{R} \times \mathbb{R}$,

$\tilde{p}_{1}\left(\tilde{f}^{q}(\tilde{\theta}, r)\right)=\tilde{\theta}+p \Rightarrow\left\{\begin{array}{l}h^{-}(\widetilde{\theta}) \leqq r \leqq h^{+}(\widetilde{\theta}) \\ \tilde{p}_{2}\left(\tilde{f}^{q}\left(\tilde{\theta}, h^{+}(\tilde{\theta})\right)\right) \leqq \tilde{p}_{2}\left(\tilde{f}^{q}(\tilde{\theta}, r)\right) \leqq \tilde{p}_{2}\left(\tilde{f}^{q}\left(\tilde{\theta}, h^{-}(\widetilde{\theta})\right)\right) .\end{array}\right.$
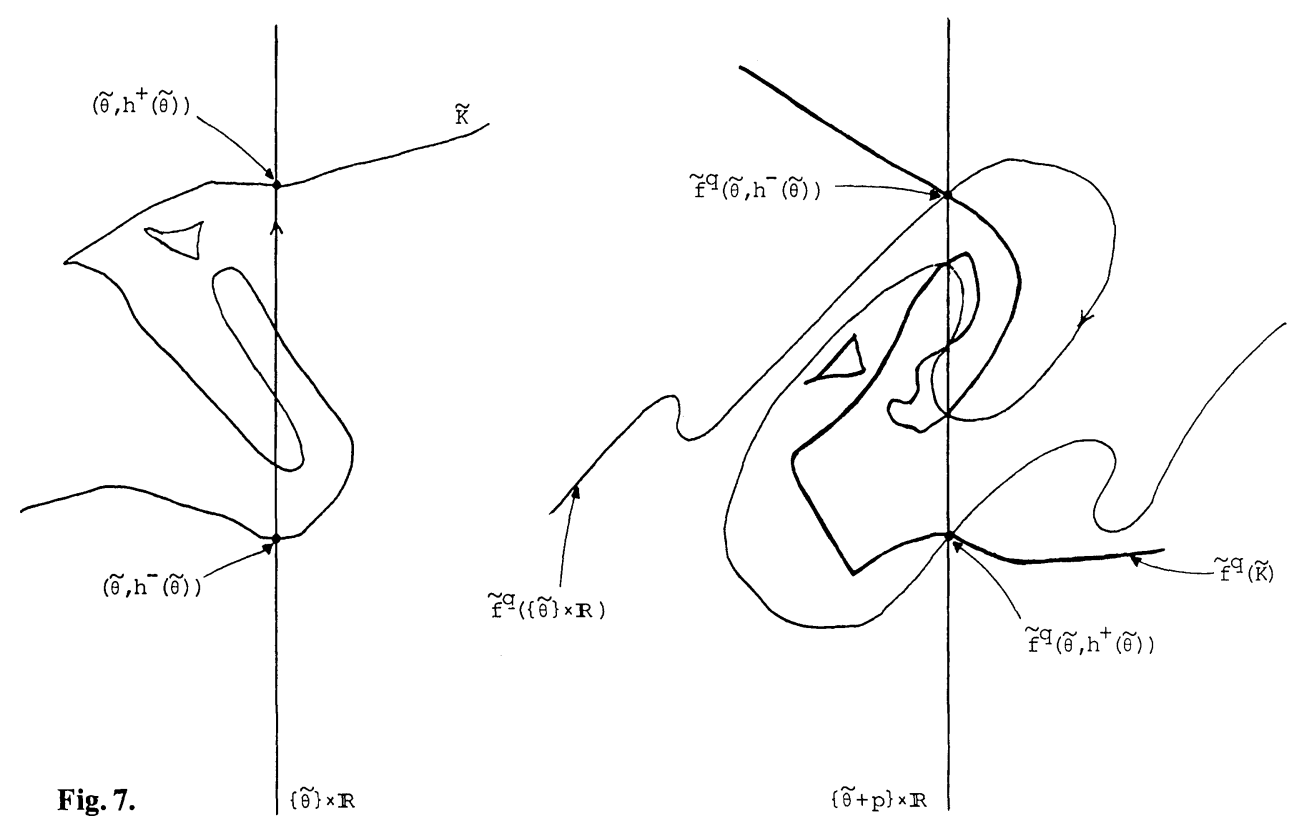
Soit $L \subset K$ un compact connexe séparant $\mathbb{T}^{1} \times \mathbb{R}$. D'après la propriété $(Q)$, si $\tilde{\theta} \in \mathbb{R}$, les courbes $\{\tilde{\theta}\} \times]-\infty, h^{-}(\tilde{\theta})\left[\right.$ et $\tilde{f}^{-q}(\{\tilde{\theta}+p\} \times] \tilde{p}_{2}\left(\tilde{f}^{q}\left(\tilde{\theta}, h^{-}(\tilde{\theta})\right)\right),+\infty[)$ ne rencontrent pas $K$. Le graphe de $h^{-}$est donc contenu dans $L$. De même, celui de $h^{+}$.

Si on suppose maintenant que, pour tout $x \in L, p_{2}\left(f^{q}(x)\right) \geqq p_{2}(x)$, on aura l'inclusion $U(L) \subset f^{q}(U(L))$.

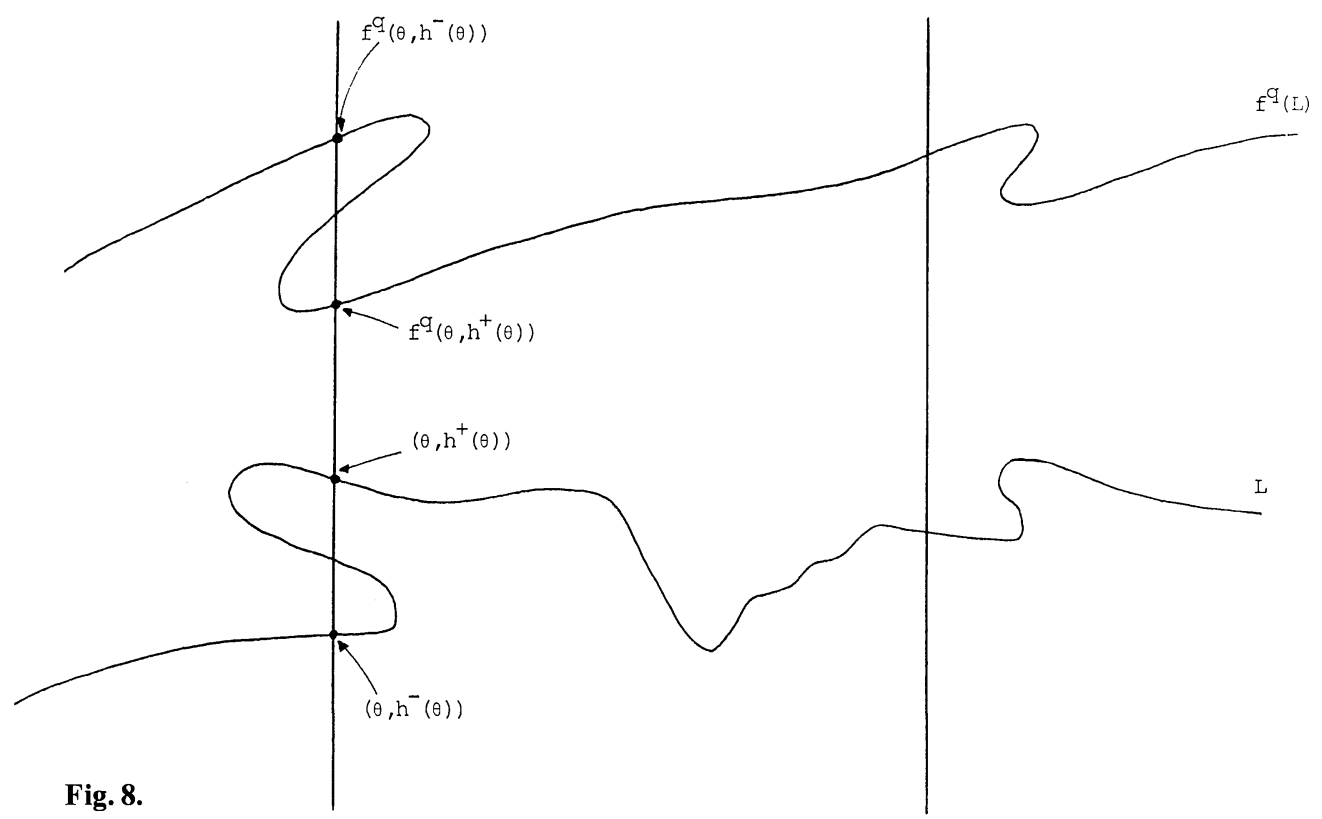

Or, $\Lambda^{\prime}$ se trouve dans tous les compacts connexes séparant $\mathbb{T}^{1} \times \mathbb{R}$, invariants par $f^{q}$. Par conséquent, $\Lambda^{\prime} \cap U(L)=\emptyset$.

De même, si, pour tout $x \in L, p_{2}\left(f^{q}(x)\right) \leqq p_{2}(x)$, on aura $\Lambda^{\prime} \cap V(L)=\emptyset$. Or, si $\tilde{x}_{0} \in \tilde{\Lambda}^{\prime-}$, on a $\tilde{p}_{1}\left(\tilde{f}^{-q}\left(\tilde{x}_{0}\right)\right)>\tilde{p}_{1}\left(\tilde{x}_{0}\right)-p$ puisque $\tilde{\varrho}^{-}<\frac{p}{q}$, et $\tilde{p}_{1}\left(\tilde{f}^{-q}\left(\tilde{y}_{0}\right)\right)>\tilde{p}_{1}\left(\tilde{y}_{0}\right)-p$ pour tout $\tilde{y}_{0}=\left(\tilde{\theta}_{0}, r_{0}\right) \in U^{-}\left(\Lambda^{\prime}\right)$. Par conséquent, la courbe $\tilde{f}^{-q}\left(\left\{\tilde{\theta}_{0}\right\} \times\right]-\infty$, $\left.\left.\tilde{p}_{2}\left(\tilde{x}_{0}\right)\right]\right)$ ne rencontre pas $\tilde{L}$, donc $\tilde{f}^{-q}\left(\tilde{x}_{0}\right) \in \tilde{\Lambda}^{\prime} \cap \tilde{U}(L)$. De même, $\tilde{\Lambda}^{\prime} \cap \tilde{V}(L) \neq \emptyset$. Le lemme découle de la connexité de $L$.

Fin de la démonstration de la Proposition 1. Dans le cas $q=1, K$ est un graphe continu et le lemme s'applique directement à $K$; mais, si $q>1, K$ peut ne plus être connexe et il faut trouver un compact connexe dans $K$ séparant $\mathbb{T}^{1} \times \mathbb{R}$. On considère pour cela l'ouvert

$$
O_{1}=\left\{x \in \mathbb{T}^{1} \times \mathbb{R} \mid \tilde{p}_{1}\left(\tilde{f}^{q}(\tilde{x})\right)<\tilde{p}_{1}(\tilde{x})+p \text { où } \pi(\tilde{x})=x\right\}
$$

qui contient $\left.\mathbb{T}^{1} \times\right]-\infty,-M\left[\right.$ et ne rencontre pas $\left.\mathbb{T}^{1} \times\right] M,+\infty[$ dès que $M$ est assez grand. On note alors $O_{2}$ la composante connexe de $O_{1}$ contenant $\left.\mathbb{T}^{1} \times\right]-\infty,-M\left[, \quad O_{3}\right.$ la composante connexe de $\mathbb{T}^{1} \times \mathbb{R}^{2} \backslash \bar{O}_{2}$ contenant $\left.\mathbb{T}^{1} \times\right] M,+\infty\left[\right.$ et $L$ la frontière de $O_{3}$. On a ainsi construit un compact connexe séparant $\mathbb{T}^{1} \times \mathbb{R}$ qui vérifie $L \subset \mathrm{FrO}_{2} \subset \mathrm{FrO}_{1} \subset K$. 
Remarque. La démonstration de la Proposition 1 s'applique aussi dans le cas d'un difféomorphisme défini sur un anneau limité par deux graphes invariants préservant l'aire et transformant chaque verticale en une courbe négative, pour montrer le théorème géométrique de Poincaré-Birkhoff.

Démonstration de la Proposition 2. Soit $\tilde{y}_{0}$ un point $\frac{p}{q}$ périodique tel que $\tilde{\varrho}^{-}<\frac{p}{q}<\tilde{\varrho}^{+}$. Si $\tilde{y}_{0} \in \tilde{U}\left(\Lambda^{\prime}\right)$, alors, d'après le Lemme 1, l'orbite de $\tilde{y}_{0}$ est formée de points de $\tilde{U}\left(\Lambda^{\prime}\right)$ non radialement accessibles.

On considère, pour tout point de discontinuité $\tilde{\theta}$ de $u^{-}$le segment $\{\widetilde{\theta}\} \times\left[u^{-}(\tilde{\theta}), \lim _{t \rightarrow \tilde{\theta}^{-}} u^{-}(t)\right]$. Il rencontre soit des points de $\tilde{U}\left(\Lambda^{\prime}\right)$ (dont l'image inverse est d'ailleurs dans $\tilde{U}^{-}\left(\Lambda^{\prime}\right)$ ), soit des points de $\Lambda^{\prime}$ accessibles. On peut donc considérer l'image par $\tilde{H}$ (définie au $\emptyset \mathrm{I}$ ) de ce segment qui est un arc continu joignant deux points de $\mathbb{R} \times\{0\}$.

Fig. 9.
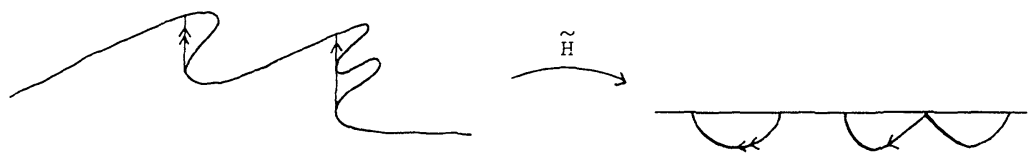

On obtient de cette façon une famille $\left(\tilde{W}_{i}\right)_{i \in I}$ au plus dénombrable d'ouverts connexes disjoints de $\mathbb{R} \times]-\infty, 0\left[\right.$, délimités par des arcs $\left(\widetilde{\Gamma_{i}}\right)_{i \in I}$ joignant deux points de $\mathbb{R} \times\{0\}$.

Fig. 10.

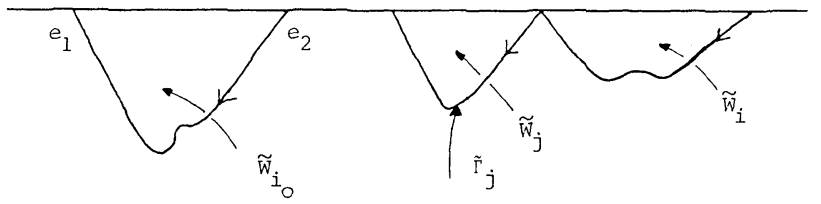

On note $\tilde{F}=\tilde{H} \circ \tilde{f} \circ \tilde{H}^{-1}$ l'homéomorphisme de $\left.\mathbb{R} \times\right]-\infty, 0[$ conjugué à $\tilde{f}$ et $\tilde{W}$ l'ouvert $\mathbb{R} \times]-\infty, 0\left[\backslash \bigcup_{i \in I} \tilde{W}_{i}=\tilde{H}\left(U^{-}\left(\Lambda^{\prime}\right)\right)\right.$. Comme $\tilde{F}^{-1}(\tilde{W}) \subset \tilde{W}$, pour tout $i \in I$, l'ouvert connexe $\tilde{F}\left(\tilde{W}_{i}\right)$ est entièrement contenu dans un des $\tilde{W}_{j}$. Soit $\tilde{Y}_{0}=\tilde{H}\left(\tilde{y}_{0}\right)$, où $\tilde{y}_{0}$ est $\frac{p}{q}$-périodique; il existe $i_{0}$ tel que $\tilde{Y}_{0} \in \bar{W}_{i_{0}}$ (en fait $\tilde{Y}_{0} \in W_{i_{0}}$ car $\tilde{F}^{-1}\left(\tilde{Y}_{0}\right) \notin \tilde{W}$ ), et comme $\tilde{F}^{q}\left(\tilde{Y}_{0}\right)=\tilde{T}^{p}\left(\tilde{Y}_{0}\right)$, on a l'inclusion $\tilde{F}^{q}\left(\tilde{W}_{i_{0}}\right) \subset \tilde{T}^{p}\left(\tilde{W}_{i_{0}}\right)$. En notant $\tilde{e}_{1}$ et $\tilde{e}_{2}$ les extrémités de $\tilde{\Gamma}_{i_{0}}$, on en déduit $\tilde{p}_{1} \circ \widetilde{\Phi}^{q}\left(\tilde{e}_{1}\right) \geqq \tilde{p}_{1} \circ \tilde{T}^{p}\left(\tilde{e}_{1}\right)$ et $\tilde{p}_{1} \circ \tilde{\Phi}^{q}\left(\tilde{e}_{2}\right) \leqq \tilde{p}_{1} \circ \tilde{T}^{p}\left(\tilde{e}_{2}\right)$ (l'application $\Phi$ a été défini au $\S \mathrm{I}$ ), ce qui entraîne $\tilde{\varrho}^{-}=\frac{p}{q}$.

Démonstration du théorème. Il suffit de prouver l'existence d'ensembles d'AubryMather dans $\Lambda^{\prime}$ pour tout nombre de rotation rationnel $\frac{p}{q}$, où $\tilde{\varrho}^{-}<\frac{p}{q}<\tilde{\varrho}^{+}$(voir $[\mathrm{K}])$. On va le montrer d'abord dans le cas où $f$ est de classe $C^{3}$, et pour cela utiliser les deux propositions et un résultat récent de Hall [Ha]. Celui-ci montre qu'un difféomorphisme de classe $C^{2}$ sur un anneau délimité par deux graphes 
invariants, déviant la verticale, admet un ensemble d'Aubry-Mather de nombre de rotation $\frac{p}{q} \in \mathbb{Q}$ dès qu'il possède un point $\frac{p}{q}$-périodique.

On choisit un anneau $\left.A=\mathbb{T}^{1} \times\right]-a,+a[(a>0)$ vérifiant $f(\bar{A}) \subset A$, et on suppose construit un difféomorphisme $g$ de classe $C^{2}$, défini sur un anneau $B=\mathbb{T}^{1} \times[-b,+b](b>a)$, coïncidant avec $f$ sur $A$, laissant invariants les deux bords $\mathbb{T}^{1} \times\{-b\}$ et $\mathbb{T}^{1} \times\{b\}$ et déviant la verticale. Le compact $\Lambda^{\prime}$ est alors invariant par $g$ et l'on a les relations $g^{-1}\left(U^{-}\left(\Lambda^{\prime}\right)\right) \subset U^{-}\left(\Lambda^{\prime}\right)$ et $g^{-1}\left(V^{+}\left(\Lambda^{\prime}\right)\right)$ $\subset V^{+}\left(\Lambda^{\prime}\right)$ puisque $f$ et $g$ coïncident sur $A$ et que $\Lambda^{\prime} \subset A$. Or, $g$ admet un ensemble d'Aubry-Mather de nombre de rotation $\frac{p}{q}$. Par une démonstration équivalente à celle du Lemme 1, on montre qu'il est formé de points non radialement accessibles; c'est donc un ensemble d'Aubry-Mather pour $f$. On utilise ensuite la Proposition 2.

Il reste à construire $g$.

On définit deux champs de vecteurs $\omega_{0}$ et $\omega_{1}$ sur $\mathbb{T}^{1} \times \mathbb{R}$ par les relations: $\omega_{0}(x)$ $=D f\left(f^{-1}(x)\right) \cdot(0,1), \omega_{1}(x)=(1, \operatorname{cotg} \beta)$ où $x \in \mathbb{T}^{1} \times \mathbb{R}$ (le réel $\beta$ est défini au $\S \mathrm{I})$.

On considère ensuite une application réelle $v$ de classe $C^{2}$, croissante et vérifiant: $v(x)=0$ si $x \leqq 0$ et $v(x)=1$ si $x \geqq 1$.

On appelle alors $\hat{\omega}$ le champ de vecteurs défini, pour tout $x=(\theta, r) \in \mathbb{T}^{1} \times \mathbb{R}$, par:

$$
\hat{\omega}(x)=(1-v(|r|-a)) \omega_{0}(x)+v(|r|-a) \omega_{1}(x) ;
$$

les équations: $\hat{g}(\theta, a)=f(\theta, a)$ et $\frac{\partial \hat{g}}{\partial r}(\theta, r)=\hat{\omega}(\hat{g}(\theta, r))$ définissent alors un difféomorphisme de classe $C^{2}$ de $\mathbb{T}^{1} \times \mathbb{R}$ sur son image, coïncidant avec $f$ sur $A$. De plus, le dessin ci-dessous prouve que l'image $\hat{g}\left(\mathbb{T}^{1} \times \mathbb{R}\right)$ est égale à $\mathbb{T}^{1} \times \mathbb{R}$. Enfin, $\hat{g}$ dévie la verticale à droite.

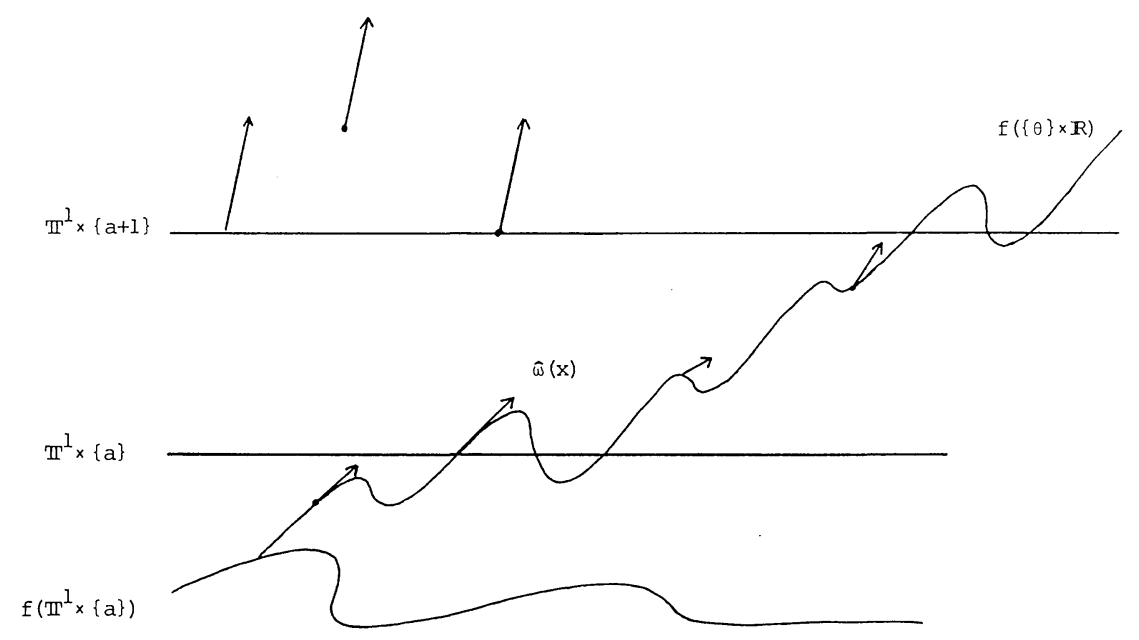

Fig. 11. 
Les images par $\hat{g}$ des droites $\{\theta\} \times \mathbb{R}$ sont, dans le revêtement $\mathbb{R} \times \mathbb{R}$, des graphes de pente égale à $\operatorname{cotg} \beta$ sauf sur un intervalle borné. Il est facile ensuite de construire un champ de vecteurs $\omega$ de classe $C^{2}$ en conservant en tout point la direction du champ $\hat{\omega}$, et le champ $\hat{\omega}$ lui-même sur $\mathbb{T}^{1} \times[-a-1, a+1]$, pour que le difféomorphisme $g$, défini par les équations $g(\theta, a)=f(\theta, a)$ et $\frac{\partial g}{\partial r}(\theta, r)=\omega(g(\theta, r))$, laisse invariantes les courbes $\mathbb{T}^{1} \times\{-b\}$ et $\mathbb{T}^{1} \times\{b\}$ où $b$ est grand, et vérifie ainsi toutes les conditions requises.

On suppose maintenant que $f$ est seulement de classe $C^{1}$ et on choisit un anneau $\left.A=\mathbb{T}^{1} \times\right]-a,+a[$ vérifiant $f(\bar{A}) \subset A$. On construit aisément - par exemple par une méthode utilisant des champs de vecteurs sur $\mathbb{T}^{1} \times \mathbb{R}$ analogue à celle que l'on vient de voir - une suite $\left(f_{n}\right)_{n \in \mathbb{N}}$ de difféomorphismes de classe $C^{3}$ de $\mathbb{T}^{1} \times \mathbb{R}$, déviant la verticale, dissipatifs, convergeant uniformément vers $f$ sur $A$ et vérifiant $f_{n}(\bar{A}) \subset A$ pour tout $n \in \mathbb{N}$. On note alors $\Lambda_{n}^{\prime}$ l'attracteur de Birkhoff de $f_{n}$ et, considérant une suite $\left(\tilde{f}_{n}\right)_{n \in \mathbb{N}}$ formée de relèvements des $f_{n}$ qui converge uniformément vers $\tilde{f}$ sur $\tilde{A}$, on note $\tilde{\varrho}_{n}^{-}$et $\tilde{\varrho}_{n}^{+}$les nombres de rotation respectivement inférieur et supérieur de $\Lambda_{n}^{\prime}$ pour $\widetilde{f}_{n}$.

Pour tout $n \in \mathbb{N}$, on a l'inclusion $\Lambda_{n}^{\prime} \subset A$. Ainsi, la suite $\left(\Lambda_{n}^{\prime}\right)_{n \in \mathbb{N}}$ est une suite bornée pour la distance de Hausdorff. Toute valeur d'adhérence de cette suite sera alors compacte, connexe, invariante par $f$ et séparera $\mathbb{T}^{1} \times \mathbb{R}$, elle contiendra donc $\Lambda^{\prime}$.

De la double inégalité suivante, vraie pour tout $k \in \mathbb{N}$, pour tout $n \in \mathbb{N}$ et pour tout $\tilde{x} \in \tilde{\Lambda}_{n}^{\prime}$ :

$$
-1+k \tilde{\varrho}_{n}^{-} \leqq \tilde{p}_{1} \circ \tilde{f}_{n}^{k}(\tilde{x})-\tilde{p}_{1}(\tilde{x}) \leqq 1+k \tilde{\varrho}_{n}^{+},
$$

on en déduit la suivante, pour tout $k \in \mathbb{N}$ et tout $x \in \Lambda^{\prime}$ :

$$
-1+k \limsup _{n \rightarrow+\infty} \tilde{\varrho}_{n}^{-} \leqq \tilde{p}_{1} \circ \tilde{f}^{k}(\tilde{x})-\tilde{p}_{1}(\tilde{x}) \leqq 1+k \liminf _{n \rightarrow+\infty} \tilde{\varrho}_{n}^{+} .
$$

En choisissant $x$ dans un ensemble d'Aubry-Mather formé de points radialement accessibles, on obtient:

$$
\limsup _{n \rightarrow+\infty} \tilde{\varrho}_{n}^{-} \leqq \tilde{\varrho}^{-} \leqq \tilde{\varrho}^{+} \leqq \liminf _{n \rightarrow+\infty} \tilde{\varrho}_{n}^{+} .
$$

Si on choisit maintenant $p / q \in] \tilde{\varrho}^{-}, \tilde{\varrho}^{+}[$, la relation précédente montre qu'il existe $N \in \mathbb{N}$ et une suite $\left(\Xi_{n}\right)_{n \geqq N}$ d'ensembles d'Aubry-Mather pour $\tilde{f}_{n}$ de nombre de rotation $p / q$ contenus dans $\Lambda_{n}^{\prime}$ et donc dans $A$. On obtient alors un ensemble d'Aubry-Mather pour $f$ en choisissant une valeur d'adhérence de cette suite.

Remarques. C'est une «bonne propriété d'intersection» vérifiée par $\Lambda^{\prime}$ qui a permis de montrer la Proposition $1\left(U(L) \subset f^{q}(U(L)) \Rightarrow \Lambda^{\prime} \cap U(L)=\emptyset\right)$. On peut montrer que le théorème est faux dans le cas d'un compact connexe quelconque séparant $\mathbb{T}^{1} \times \mathbb{R}$ invariant par $f$. Il peut manquer des nombres de rotation entre $\tilde{\varrho}_{A}^{-}$et $\tilde{\varrho}_{A}^{+}$. Supposons en effet que l'application $\tilde{f}_{0}$ (définie au $\S$ I) possède de plus un point $\frac{p}{q}$-périodique hyperbolique au-dessus de $C^{+}$et que $\tilde{\varrho}_{0}^{+}$(voir $\S$ I) est irrationnel. On peut montrer l'inégalité $\tilde{\varrho}_{A(\varepsilon, A)}^{+}<\tilde{\varrho}_{0}^{+}$où $\tilde{\varrho}_{A(\varepsilon, A)}^{+}$est le nombre de rotation supérieur (pour $f_{\varepsilon}$ ) de l'ensemble $\Lambda(\varepsilon, A)=\bigcap_{n \in \mathbb{N}} f_{\varepsilon}^{n}(A)$. Si $\varepsilon$ est assez petit, $\tilde{f_{\varepsilon}}$ possède 
un point $\frac{p}{q}$-périodique et, pour un anneau $B=\mathbb{T}^{1} \times[-M,+M]$ suffisament large, on aura $f(B) \subset B, \varrho_{\Lambda(\varepsilon, B)}^{+} \geqq \frac{p}{q}$, et finalement:

$$
\tilde{\varrho}_{\Lambda(\varepsilon, B)}^{-} \leqq \tilde{\varrho}_{\Lambda(\varepsilon, A)}^{-} \leqq \tilde{\varrho}_{\Lambda(\varepsilon, A)}^{+}<\tilde{\varrho}_{0}^{+}<\frac{p}{q} \leqq \tilde{\varrho}_{\Lambda(\varepsilon, B)}^{+} \text {. }
$$

Il est alors facile de montrer que $\tilde{f}_{\varepsilon}$ n'a pas d'ensemble d'Aubry-Mather de nombre de rotation dans $] \tilde{\varrho}_{\Lambda(\varepsilon, A)}^{+}, \tilde{\varrho}_{0}^{+}[$.

Remerciements. Je tiens à remercier Michel Herman de m'avoir proposé d'étudier les attracteurs de Birkhoff. Je remercie également Alain Chenciner et Daniel Bennequin pour leurs précieux conseils et leur incitation à simplifier de nombreux détails de la démonstration, ainsi que Raphaël Douady pour sa lecture attentive du texte. Je n'oublie pas non plus Marie-Jo Lécuyer pour la gentillesse et le soin avec lesquels elle a déssiné les figures.

\section{Bibliographie}

[B1] Birkhoff, G.D.: Sur quelques courbes fermées remarquables. Bull. Soc. Math. Fr. 60, 1-26 (1932); aussi dans Collected Math. Papers of G. D. Birkhoff, Vol. II, p. 418-443, New York: Dover 1968

[B2] Birkhoff, G.D.: Sur l'existence de régions d'instabilité en dynamique, Ann. Inst. Henri Poincaré 8 (1932) et Collected Math. Papers, Vol.II, p. 444-461

[Cha] Charpentier, M.: Sur quelques propriétés des courbes de M. Birkhoff. Bull. Soc. Math. Fr. 62, 193-224 (1934)

[Che] Chenciner, A.: Séminaire Bourbaki, ${ }^{\circ}$ 622. Astérisque, 121-122, Soc. Math. de France 147-170 (1985)

[Ha] Hall, G.R. A topological version of a theorem of Mather on twist maps. Ergodic Theory Dyn. Syst. 4, 585-603 (1984)

[He] Herman, M.: Sur les courbes invariantes par les difféomorphismes de l'anneau. Astérisque 103-104, Soc. Math. de France (1983)

[K] Katok, A.: Some remarks on Birkhoff and Mather twist map theorem. Ergodic Theory Dyn. Syst. 185-192 (1982)

Communicated by J. Mather

Reçu le 15 avril 1985; revisé le 25 avril 1986 